\title{
Vasoactive Therapy in Systemic Sclerosis: Real-life Therapeutic Practice in More Than 3000 Patients
}

\author{
Pia Moinzadeh, Gabriela Riemekasten, Elise Siegert, Gerhard Fierlbeck, Joerg Henes, \\ Norbert Blank, Inga Melchers, Ulf Mueller-Ladner, Marc Frerix, Alexander Kreuter, \\ Christian Tigges, Nina Lahner, Laura Susok, Claudia Guenther, Gabriele Zeidler, \\ Christiane Pfeiffer, Margitta Worm, Sigrid Karrer, Elisabeth Aberer, Agnes Bretterklieber, \\ Ekkehard Genth, Jan C. Simon, Joerg H.W. Distler, Ruediger Hein, Matthias Schneider, \\ Cornelia S. Seitz, Claudia Herink, Kerstin Steinbrink, Miklos Sárdy, Rita Varga, \\ Hartwig Mensing, Christian Mensing, Percy Lehmann, Gunther Neeck, Christoph Fiehn, \\ Manfred Weber, Matthias Goebeler, Harald Burkhardt, Michael Buslau, Keihan Ahmadi-Simab, \\ Andrea Himsel, Aaron Juche, Ina Koetter, Annegret Kuhn, Michael Sticherling, \\ Martin Hellmich, Kathrin Kuhr, Thomas Krieg, Jan Ehrchen, Cord Sunderkoetter, \\ and Nicolas Hunzelmann, for the German Network for Systemic Scleroderma
}

\begin{abstract}
Objective. Vasculopathy is a key factor in the pathophysiology of systemic sclerosis (SSc) and the main cause for Raynaud phenomenon (RP), digital ulcers (DU), and/or pulmonary arterial hypertension (PAH). It is so far unknown how patients with SSc are treated with vasoactive agents in daily practice. To determine to which extent patients with SSc were treated with different vasoactive agents, we used data from the German Network for Systemic Scleroderma registry.

Methods. The data of 3248 patients with SSc were analyzed.

Results. Patients were treated with vasoactive drugs in $61.1 \%$ of cases (1984/3248). Of these, $47.6 \%$ received calcium channel inhibitors, followed by $34.2 \%$ treated with angiotensin-converting enzyme (ACE) inhibitors, $21.1 \%$ treated with intravenous (IV) prostanoids, $10.1 \%$ with pentoxifylline, $8.8 \%$ with angiotensin 1 receptor antagonists (AT1RA), 8.7\% with endothelin 1 receptor antagonists (ET1RA), $4.1 \%$ with phosphodiesterase type 5 (PDE5) inhibitors, and 5.3\% with others. Patients with RP received vasoactive therapy in $63.3 \%$ of cases, with DU in $70.1 \%$, and with PAH in $78.2 \%$ of cases. Logistic regression analysis revealed that patients with PAH were significantly more often treated with PDE5 inhibitors and ET1RA, and those with DU with ET1RA and IV prostanoids. In addition, $41.8 \%$ of patients were treated with ACE inhibitors and/or AT1RA. Patients registered after 2009 received significantly more often ET1RA, AT1RA, and IV prostanoids compared with patients registered prior to 2005 .

Conclusion. These data clearly indicate that many patients with SSc do not yet receive sufficient vasoactive therapy. Further, in recent years, a marked change of treatment regimens can be observed. (First Release November 15 2015; J Rheumatol 2016;43:66-74; doi:10.3899/jrheum.150382)
\end{abstract}

Key Indexing Terms:

SYSTEMIC SCLEROSIS VASOACTIVE THERAPY

REAL LIFE GERMAN NETWORK FOR SYSTEMIC SCLERODERMA

\begin{abstract}
From the Department of Dermatology, University Hospital of Cologne; Department of Internal Medicine, Hospital of Cologne Merheim; Institute of Medical Statistics, Informatics and Epidemiology, University of Cologne, Cologne; Department of Rheumatology, University Medical Center Schleswig-Holstein, Lübeck; Department of Rheumatology and Clinical Immunology, and Department of Dermatology, University of Berlin, Charité, Berlin; Department of Rheumatology, Immanuel Hospital, Berlin-Buch; Department of Dermatology, and Department of Rheumatology, University Hospital Tübingen, Tübingen; Department of Internal Medicine, Division of Rheumatology, Heidelberg University Hospital; Division of Immunogenetics, German Cancer Research Center, Heidelberg; Department of Rheumatology and Clinical Immunology, University Medical Center, Freiburg; Department of Rheumatology and Clinical Immunology, University of Giessen, Kerckhoff Clinic, Bad Nauheim; Department of Dermatology, HELIOS St. Elisabeth Clinic,
\end{abstract}

\begin{abstract}
Oberhausen; Department of Dermatology, St. Joseph Hospital, RuhrUniversity of Bochum, Bochum; Department of Dermatology, University Hospital Carl Gustav Carus, Dresden; Department of Rheumatology, Johanniter-Hospital, Treuenbrietzen; Department of Dermatology, Ulm University Hospital, Ulm; Department of Dermatology, University Hospital Regensburg, Regensburg; Department of Rheumatology, Clinic of Rheumatology of Aachen, Aachen; Department of Dermatology, University Hospital Leipzig, Leipzig; Department of Rheumatology, and Department of Dermatology, University Hospital Erlangen, Erlangen; Department of Dermatology, TUM University Hospital; Department of Dermatology, Ludwig Maximilian University, Munich; Department of Rheumatology and Nephrology, University Hospital Duesseldorf, Duesseldorf; Department of Dermatology, University Hospital Göttingen, Göttingen; Department of Dermatology, University Medical Center, Johannes Gutenberg University, Mainz; Clinic for Dermatology, Hamburg
\end{abstract}


Alstertal; Department of Dermatology, Helios Clinic, Wuppertal; Center for Rheumatology Bad Doberan, Bad Doberan; Center for Rheumatology Acura, Baden-Baden; Department of Dermatology, University Hospital of Würzburg, Würzburg; Department of Rheumatology, University Hospital Frankfurt, Frankfurt; Center for Rheumatology (Rehabilitation), Rheinfelden; Clinic for Internal Medicine, Klinikum Stephansplatz; Department of Rheumatology, Asklepios Clinic Altona, Hamburg; Department of Rheumatology, GRP-Hospital Ruesselheim, Darmstadt; Department of Dermatology and Venereology, University Hospital Muenster, Muenster, Germany; Department of Dermatology, Medical University of Graz, Graz, Austria.

Supported by a grant of the German Federal Ministry of Education and Research (BMBF; 01GM0310 NH, TK; 01GM0631 CS) and the Edith Busch Foundation. The work of Pia Moinzadeh was supported by "Koeln Fortune" (155/2014) and "Deutsche Stiftung Sklerodermie" (3649/0096/31) grants.

P. Moinzadeh, MD, Department of Dermatology, University Hospital of Cologne; G. Riemekasten, MD, Department of Rheumatology, University Medical Center Schleswig-Holstein; E. Siegert, Department of Rheumatology and Clinical Immunology, University of Berlin, Charité; G. Fierlbeck, MD, Department of Dermatology, University Hospital Tübingen; J. Henes, MD, Department of Rheumatology, University Hospital Tübingen; N. Blank, MD, Department of Internal Medicine, Division of Rheumatology, Heidelberg University Hospital; I. Melchers, MD, Department of Rheumatology and Clinical Immunology, University Medical Center; U. Mueller-Ladner, MD, Department of Rheumatology and Clinical Immunology, University of Giessen, Kerckhoff Clinic; M. Frerix, MD, Department of Rheumatology and Clinical Immunology, University of Giessen, Kerckhoff Clinic; A. Kreuter, MD, Department of Dermatology, HELIOS St. Elisabeth Clinic; C. Tigges, MD, Department of Dermatology, HELIOS St. Elisabeth Clinic; N. Lahner, MD, Department of Dermatology, St. Joseph Hospital, Ruhr-University of Bochum; L. Susok, MD, Department of Dermatology, St. Joseph Hospital, Ruhr-University of Bochum; C. Guenther, MD, Department of Dermatology, University Hospital Carl Gustav Carus; G. Zeidler, MD, Department of Rheumatology, Johanniter-Hospital; C. Pfeiffer, MD, Department of Dermatology, Ulm University Hospital; M. Worm, MD, Department of Dermatology, University of Berlin, Charité; S. Karrer, MD, Department of Dermatology, University Hospital Regensburg; E. Aberer, MD, Department of Dermatology, Medical University of Graz; A.

Bretterklieber, MD, Department of Dermatology, Medical University of Graz; E. Genth, MD, Department of Rheumatology, Clinic of Rheumatology of Aachen; J.C. Simon, MD, Department of Dermatology, University Hospital Leipzig; J.H. Distler, MD, Department of Rheumatology, University Hospital Erlangen; R. Hein, MD, Department of Dermatology, TUM University Hospital; M. Schneider, MD, Department of Rheumatology and Nephrology, University Hospital Duesseldorf; C.S. Seitz, MD, Department of Dermatology, University Hospital Göttingen; C. Herink, MD, Department of Dermatology, University Hospital Göttingen; K. Steinbrink, MD, Department of Dermatology, University Medical Center, Johannes Gutenberg University, M. Sárdy, MD, Department of Dermatology, Ludwig Maximilian University; R. Varga, MD, Department of Dermatology, Ludwig Maximilian University; H. Mensing, MD, Clinic for Dermatology; C. Mensing, MD, Clinic for Dermatology; P. Lehmann, MD, Department of Dermatology, Helios Clinic; G. Neeck, MD, Center for Rheumatology Bad Doberan; C. Fiehn, MD, Center for Rheumatology Acura; M. Weber, MD, Department of Internal Medicine, Hospital of Cologne Merheim; M. Goebeler, MD, Department of Dermatology, University Hospital of Würzburg; H. Burkhardt, MD, Department of Rheumatology, University Hospital Frankfurt; M. Buslau, MD, Center for Rheumatology

(Rehabilitation); K. Ahmadi-Simab, MD, Clinic for Internal Medicine, Klinikum Stephansplatz; A. Himsel, MD, Department of Rheumatology, GRP-Hospital Ruesselheim; A. Juche, MD, Department of Rheumatology, Immanuel Hospital; I. Koetter, MD, Department of Rheumatology, Asklepios Clinic Altona; A. Kuhn, MD, Division of Immunogenetics, German Cancer Research Center; M. Sticherling, MD, Department of Dermatology, University Hospital Erlangen; M. Hellmich, MD, Institute of Medical Statistics, Informatics and Epidemiology, University of Cologne; K. Kuhr, MD, Institute of Medical Statistics, Informatics and Epidemiology, University of Cologne; T. Krieg, MD, Department of Dermatology, University Hospital of Cologne; J. Ehrchen, MD,
Department of Dermatology and Venereology, University Hospital Muenster; C. Sunderkoetter, MD, Department of Dermatology and Venereology, University Hospital Muenster; N. Hunzelmann, MD, Department of Dermatology, University Hospital of Cologne.

Address correspondence to Dr. P. Moinzadeh, Department of Dermatology and Venereology, University of Cologne, Kerpener Str. 62, 50937 Cologne, Germany.E-mail: pia.moinzadeh@uk-koeln.de

Full Release Article. For details see Reprints and Permissions at jrheum.org Accepted for publication August 28, 2015.

Vasculopathy presents as a major clinical problem in patients with systemic sclerosis ( $\mathrm{SSc}$ ) and is the main cause for Raynaud phenomenon (RP), digital ulcerations (DU), pulmonary hypertension, telangiectasia and/or renovascular complications with proteinuria, and severe renal crisis ${ }^{1,2}$.

The pathophysiology of vasculopathy in SSc is complex and only partially understood. It is a result of an imbalance between vasoconstrictors and vasodilators. Endothelial cell damage is one of the early events in SSc, although it is still not definite whether this is also the initiating factor in this multisystem disease ${ }^{3}$.

Unfortunately, no curative treatment strategies are available yet; however, depending on organ manifestations, several vasodilator agents are available to reverse vasoconstriction ${ }^{4}$. These drugs are known to enhance vasodilation and reduce vasoconstriction, and some are known to reduce endothelial cell injury.

In 2009, the European League Against Rheumatism (EULAR) Scleroderma Trials and Research group (EUSTAR) published recommendations for the management of organ manifestations in patients with SSc, including vasoactive treatment options ${ }^{5,6}$. Evidence-based recommendations have also been published by members of the German Network for Systemic Scleroderma (DNSS) for the treatment of $\mathrm{RP}^{7}$ and for the management of $\mathrm{DU}^{8}$. EUSTAR recommended calcium channel inhibitors (CCI) as one of the first-line treatment options for $\mathrm{RP}^{5,9}$. In addition, the EULAR expert group included endothelin 1 receptor antagonists (ET1RA, Bosentan) for digital vasculopathy and pulmonary arterial hypertension (PAH). The European Medicines Agency has approved this drug for the treatment of PAH and the prevention of recurrent DU $6,10,11,12$. Other ET1RA (ambrisentan, macitentan) are approved for the treatment of PAH only ${ }^{13,14,15}$.

Intravenously (IV) applied prostanoids such as iloprost are the gold standard for patients with active $\mathrm{DU}^{5}$. It has been reported that iloprost improves RP as well as the healing of already existing DU, reduces the frequency, duration, and severity of RP attacks, and inhibits the formation of new ulcers, with a beneficial effect beyond 9 weeks ${ }^{16,17,18}$.

Angiotensin-converting enzyme (ACE) inhibitors ${ }^{19}$, as well as angiotensin 1 receptor antagonists (AT1RA) ${ }^{20}$, have been used to encourage blood flow and improve clinical features [RP, proteinuria, renal function, hypertension (HTN), gastrointestinal symptoms] associated with vasculopathy ${ }^{21}$. ACE inhibitors are known to have an effect on the endothelial 
function and vascular remodeling ${ }^{19}$, while AT1RA may have also an antifibrotic effect beside the vasodilative action ${ }^{20}$.

Phosphodiesterase type 5 (PDE5) inhibitors are approved for patients with erectile dysfunction as well as PAH, acting through guanosine 3',5'-cyclic monophosphate. However, a single-center pilot study using sildenafil in patients with therapy-refractory $\mathrm{DU}^{22}$, as well as a prospective, open-label, uncontrolled pilot study using sildenafil for $\mathrm{PAH}^{23}$, indicated a significant improvement in terms of severity and frequency of $\mathrm{RP}^{24,25}$ and DU.

SSc is a very rare and heterogeneous disease in which patients are treated by several subspecialties. This is often associated with varying therapeutic approaches. There are no data on the use of vasoactive therapy for patients with SSc in a real-life setting. We therefore wanted to determine which vasoactive agents are used and whether treatment approaches change over time.

\section{MATERIALS AND METHODS}

The database of the DNSS, funded by the Federal Ministry of Education and Research, was set up in October 2003 with the aim of improving clinical care, diagnosis, followup, and treatment of patients presenting with different disease manifestations. Currently, there are more than 40 clinical centers involved, consisting of different subspecialties such as rheumatology, dermatology, pulmonology, and nephrology. The database holds data of more than 3200 patients. The 4-page, disease- and organ-specific questionnaire collects a core set of clinical data to determine the current status of the disease, including information on sex, date of birth, and onset of organ manifestations, as well as current symptoms, with characteristic laboratory data such as antinuclear antibodies, as described ${ }^{26}$. Further, information on current therapy including corticosteroid dosage, immunosuppressive treatment ${ }^{27}$, and the use of vasoactive drugs is part of the DNSS questionnaire. Followup visits and investigations (echocardiography, electrocardiogram, lung function test, etc.) are recommended at least once per year, depending on the severity of organ manifestations and the course of the disease. Data ranging from 2003 through 2013 were analyzed.

Symptom characteristics for vascular involvement. RP was defined by repeated vasospasms of small digital arterioles/arteries at fingers and/or toes, usually triggered by cold or emotional stress. Digital tip ischemia/ulcer were defined as digital pitting scars, ulcerations or gangrene, or both located at finger and/or toe tips.

PAH was verifiable in case of clinical evidence of right heart failure and/or increased mean pulmonary arterial pressure (PAPm $>25 \mathrm{mmHg}$ at rest or PAP $30 \mathrm{mmHg}$ during exercise), determined by right heart catheterization. Echocardiography was performed to identify likely PAH (estimated right ventricular systolic pressure $>40 \mathrm{mmHg}$ ). Patients were diagnosed with cardiac disease if palpitation, conduction disturbance, and/or diastolic dysfunction was detectable. Kidney involvement was defined as renal insufficiency encompassing renal insufficiency due to acute renal crisis (creatinine clearance age-related $<80 \mathrm{ml} / \mathrm{min}$ ) or proteinuria as defined by albuminuria $\geq 30 \mathrm{mg} / 24 \mathrm{~h}$ or $\geq 20 \mathrm{mg} / \mathrm{l}$; proteinuria $\geq 300 \mathrm{mg} / 24 \mathrm{~h}$ or $\geq 200$ $\mathrm{mg} / \mathrm{l}^{26}$.

The Ethics Committee of the coordinating center (i.e., the Cologne University Hospital) approved the patient information and consent form of the DNSS registry, with which all participating centers sought the approval of their local ethics committees prior to registering patients. To participate in the study, all patients provided written informed consent.

Data distributions were summarized by number and percentage. Association of prescription data, clinical data, and time were evaluated by cross tabulation (Fisher's exact test) and multivariable logistic regression (backward, stepwise selection procedure; variables considered: age, sex, RP,
PAH, DU, HTN). For logistic regression models, OR, corresponding $95 \%$ $\mathrm{CI}$, and $\mathrm{p}$ values (Wald test) were given. To guard against Type I error inflation resulting from multiple testing, only $\mathrm{p}$ values below 0.001 should be considered to indicate statistical significance. Because the amount of missing data was small $(<10 \%)$, analyses were based on complete cases. Calculations and figures were carried out using SPSS (IBM Corp.) and Excel (Microsoft Corp.).

\section{RESULTS}

From 2003 to 2013, a total of 3248 patients diagnosed with SSc have been registered in the database of the DNSS. Of these, $47.9 \%(1555 / 3248)$ were diagnosed as limited SSc (lcSSc), $30.0 \%(976 / 3248)$ as diffuse SSc (dcSSc), 10.0\% (325/3248) as SSc-overlap syndromes, $8.2 \%(267 / 3248)$ as undifferentiated SSc, and $0.7 \%(23 / 3248)$ as SSc sine scleroderma, as described ${ }^{26,27,28}$ (Table 1).

Information on the application of vasoactive drugs existed in $95.6 \%(3104 / 3248)$ of cases $(144,4.4 \%$ data were missing), including information on the use of ACE inhibitors as well as other vasodilative agents, e.g., CCI, ET1RA, PDE5 inhibitors, $\alpha$-blockers, AT1RA, and prostanoids, as well as topical treatment (nitrates).

Clinical characteristics and information on their vasoactive treatment were evaluated at the first visit and during all followups. The duration of the followup visits was variable among registered patients depending on the severity of organ manifestations. Information on clinical features and therapy was available for 1785 patients after their second followup visit (median \pm SD followup time between first visit and second followup $1.1 \pm 1.2 \mathrm{yrs}$ ), followed by 1206 patients after the third (median \pm SD followup time between first visit and third followup $2.3 \pm 1.3 \mathrm{yrs}$ ), 849 patients after the fourth (median \pm SD followup time between first visit and fourth followup $3.3 \pm 1.6 \mathrm{yrs}), 571$ after the fifth $(4.3 \pm 1.7 \mathrm{yrs})$, and 371 after the sixth $(5.3 \pm 1.7 \mathrm{yrs})$.

Of 3248 patients, $61.1 \%(\mathrm{n}=1984)$ were treated with vasoactive agents, and $63.3 \%$ (1255/1984) of patients treated with vasoactive drugs received just 1 drug compared with $28.0 \%(556 / 1984)$ who received more than 1 vasodilative drug (7.7\%; missing data).

Among these patients (initial visit, inclusion into the registry), $47.6 \%(945 / 1984)$ received CCI, followed by $34.2 \%(678 / 1984)$ treated with ACE inhibitors, $21.1 \%$ (419/1984) treated with IV prostanoids (iloprost, alprostadil), $10.1 \%$ (201/1984) with pentoxifylline, $8.8 \%$ (175/1984) with AT1RA, 8.7\% (173/1984) with ET1RA, 4.1\% (82/1984) with PDE5 inhibitors, 2.4\% (48/1984) with topical vasodilative agents, $1.0 \%$ with $\alpha$-blocker, and $1.9 \%$ (38/1984) with other medications, including moxonidine, nitrates, and serotonin reuptake inhibitors (Table 1 ).

The overall frequency of vasoactive treatment did not differ significantly among the 3 major SSc subsets (e.g., $65.9 \%$ of $1 \mathrm{cSSc}, 66.3 \%$ of $\mathrm{dcSSc}$, and $57.8 \%$ of the SSc-overlap syndromes), sex, or autoantibody status. However, patients with a modified Rodnan skin score

Personal non-commercial use only. The Journal of Rheumatology Copyright @ 2017. All rights reserved 
Table 1. Patient characteristics with frequencies of sex, SSc subsets, ANA, organ involvement, current symptoms, and vasoactive therapies together with (A) the frequency of missing data for all patients and for 2 different age groups, including (B) the frequencies as well as the p values (Fisher's exact test).

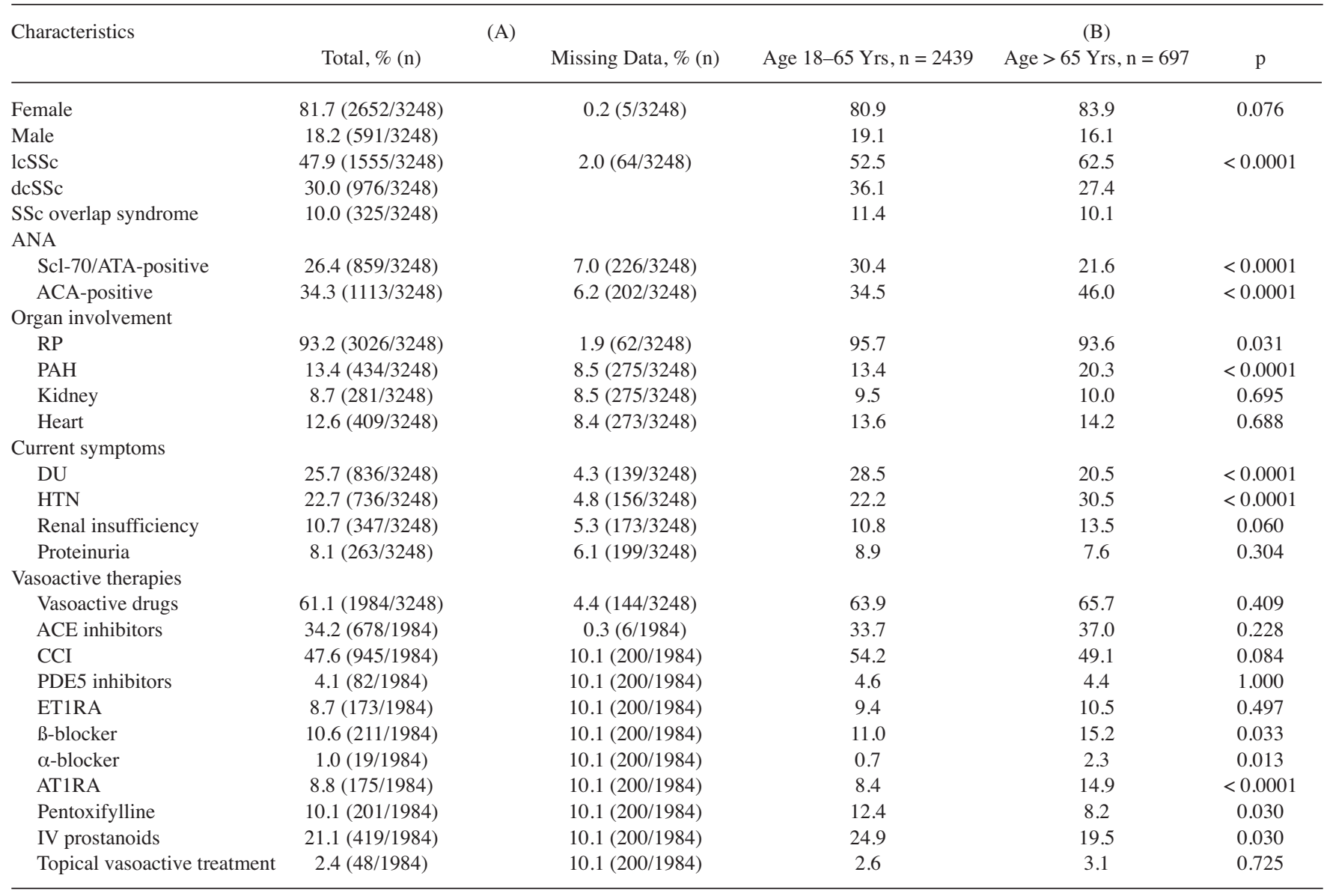

SSc: systemic sclerosis; ANA: antinuclear antibodies; lcSSc: limited cutaneous SSc; dcSSc: diffuse cutaneous SSc; ATA: antitopoisomerase antibody; ACA: anticentromere antibody; RP: Raynaud phenomenon; PAH: pulmonary arterial hypertension; DU: digital ulcers; HTN: hypertension; ACE: angiotensin-converting enzyme; CCI: calcium channel inhibitors; PDE5: phosphodiesterase type 5; ET1RA: endothelin 1 receptor antagonists; AT1RA: angiotensin 1 receptor antagonists; IV: intravenous.

(mRSS) of more than 15 received vasoactive treatment significantly more often $(70.1 \%)$ compared with those patients with an mRSS $<10(62.5 \%, \mathrm{p}=0.001)$. Further, a similar number of patients, treated by different subspecialties, received vasoactive treatment. Patients treated by rheumatologists received vasoactive treatment in $63.7 \%$ of cases compared with $64.3 \%$ of patients treated by dermatologists. Interestingly, significantly more patients registered in centers for internal medicine were treated with ET1RA $(11.4 \%$ vs $7.0 \%, \mathrm{p}=0.002)$ and PDE5 inhibitors $(6.4 \%$ vs $1.7 \%, \mathrm{p}<$ $0.0001)$, while significantly more patients seen in dermatological centers were treated with IV prostanoids $(28.3 \%$ vs $20.5 \%, \mathrm{p}<0.0001)$. These patient groups did not differ significantly in organ manifestations or clinical features, which required vasoactive treatment or reflect severity of the disease.

Vasoactive treatment depending on $R P, D U, P A H$, and the age at disease onset. Independently of concomitant manifestations, $65.1 \%$ of patients with RP received vasoactive treatment, $84.7 \%$ of patients with $\mathrm{PAH}$, and $76.4 \%$ of patients with DU $(\mathrm{p}<0.0001)$.

Focusing on those patients, who were positive for only 1 vasculopathic manifestation, significantly more patients with a history of PAH but without simultaneous DU (84.2\% vs $15.8 \%, \mathrm{p}<0.0001)$ received vasoactive therapy, which was also true for those with DU, but not simultaneously with PAH (74.7\% vs 33.2\%, p < 0.0001; Figure 1A). Of those, patients with PAH were treated more often with PDE5 inhibitors (16.4\%) and ET1RA (24.1\%), while $52.7 \%$ of patients with DU received CCI and 24\% IV prostanoids (Figure 1B).

Logistic regression analyses clearly indicated that patients diagnosed with PAH had a significantly increased chance to be treated with ET1RA(OR 6.9, 95\% CI 4.8-9.8, p < 0.001). PDE5 inhibitors were significantly more often prescribed in patients with PAH (OR 7.2, 95\% CI 4.5-11.7, p <0.001), and ET1RA significantly more often in patients with DU (OR 2.8, 95\% CI 2.0-3.9, p < 0.001). For the treatment with ACE inhibitors and/or AT1RA, independent factors were sex Personal non-commercial use only. The Journal of Rheumatology Copyright $\subset$ 2017. All rights reserved. 

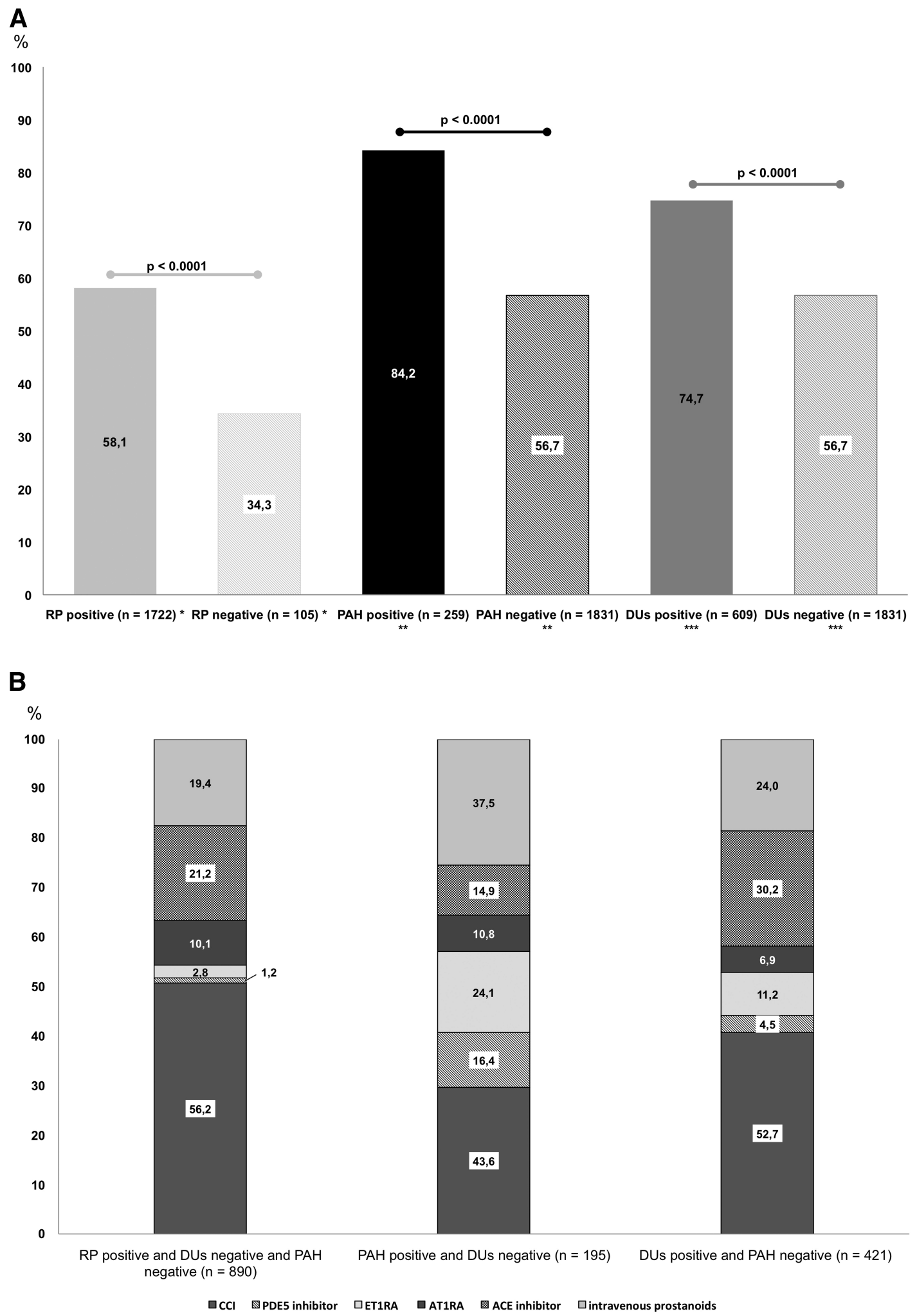

Figure 1. (A) The use of vasoactive drugs in total depending on symptoms/organ manifestations (all $\mathrm{p}<0.0001)$ and (B) the division into the most frequently used vasoactive drugs. Significant differences among the 3 groups were found for CCI, PDE5 inhibitors, ET1RA, ACE inhibitors, and IV prostanoids ( $\mathrm{p}<0.001$ ). Triple-negative patients were not considered here. * RP-positive versus negative, but without PAH and DU. ** PAH-positive versus negative, but without DU. *** DU-positive versus negative, but without PAH. CCI: calcium channel inhibitors; PDE5: phosphodiesterase type 5; ET1RA: endothelin 1 receptor antagonists; ACE: angiotensin-converting enzyme; IV: intravenous; RP: Raynaud phenomenon; PAH: pulmonary arterial hypertension; DU: digital ulcers. 
(female OR 0.6, 95\% CI 0.5-0.7, p < 0.001), age $(1.007,95 \%$ CI $1.002-1.011, \mathrm{p}=0.006)$, PAH (OR 1.8, 95\% CI 1.5-2.3, $\mathrm{p}<0.001$ ), and HTN (OR 5.4, 95\% CI 4.5-6.5, p < 0.001), whereas RP (OR 5.1, 95\% CI 1.2-21.4, $\mathrm{p}=0.026)$ and DU (OR 1.7, 95\% CI 1.4-2.2, p < 0.001) were associated with the prescription of IV prostanoids (Table 2).

Interestingly, in our patient cohort, elderly patients (age of disease onset above $65 \mathrm{yrs}$ ) had significantly more PAH (20.3\%) compared with those with an age at disease onset between 18 and 65 years $(13.4 \%, \mathrm{p}<0.0001)$. There was no significant difference in treatment strategies in both age groups (Table 1B).

Vasoactive treatment depending on the observation time. Comparing different observation periods, the overall percentage of patients receiving vasoactive therapy showed no change in treatment prior to 2005 and after 2009. However, the frequency of the use of different drug classes changed remarkably over the observation time (Table 3; Figure 2).

The number of patients receiving ACE inhibitors and/or AT1RA after 2009 compared with the number prior to 2005 increased significantly (Table 3 ). Focusing on the data of the followup visits between 2003 and 2013, the frequency of the use of ET1RA increased clearly since 2003 and for PDE5 inhibitors slightly after 2006 (Figure 2). In addition, the number of prescriptions (exemplary for RP) decreased between 2003 and 2013 for CCI and pentoxifylline (Figure 2).

When patients registered after 2009 were compared with patients registered prior to 2005, it was found that after 2009, significantly more patients received ET1RA $(12.2 \%$ vs $5.9 \%$, $\mathrm{p}<0.0001)$, AT1RA $(12.3 \%$ vs $5.4 \%, \mathrm{p}<0.0001)$, and IV prostanoids $(26.8 \%$ vs $17.7 \%, \mathrm{p}<0.001)$.

\section{DISCUSSION}

Vasculopathy as the leading cause of RP, DU, renal failure, as well as for PAH, plays a central role in morbidity and mortality of patients with SSc. In reanalyses, a number of new drugs have become available that target organ complications such as PAH and DU. Also, for the first time, consensus basal treatment recommendations were developed and published by EULAR in 2009. However, the number of controlled clinical studies showing clear evidence for amelio- rating vasculopathy in patients with SSc is still very limited ${ }^{29}$. This is clearly because of (1) the lack of clinical studies with sufficient power, (2) the varying efficacy of available targeting drugs, (3) the heterogeneity of clinical features, and (4) the varying response to different therapy approaches. A study by Pope, et al, including data from 2004 through 2011, analyzed the influence of the EULAR guidelines on treatment strategies by comparing data before and after 2009 and did not find a significant change in treatment strategies before and after the EULAR recommendations were published in $2009^{30}$.

In the light of these facts, it was of great interest and importance to us as to how vascular disease has been treated in clinical centers in Germany. Therefore, we evaluated the current application of vasoactive drugs and a potential association with organ involvement and other clinical features in patients with SSc registered at the DNSS between 2003 and 2013.

In this cohort, the most commonly prescribed vasoactive drugs were CCI (47.6\%), followed by ACE inhibitors (34.2\%) and IV prostanoids $(21.1 \%)$.

Our analysis showed that only $58.1 \%$ of patients with RP were treated with vasoactive drugs and of those, only $56.2 \%$ received CCI. Similar frequencies $(60 \%)$ were also described in the Canadian cohort ${ }^{30}$.

Patients with DU received vasodilating agents only in $74.7 \%$ of cases, and again only $24.0 \%$ of those were treated with IV prostanoids and $11.2 \%$ with ET1RA. These findings are in contrast to the data of the Canadian cohort. Because IV prostanoids are not approved for the treatment of DU in Canada, only about $5 \%$ of the patients received this drug ${ }^{30}$. According to the EULAR recommendations for the treatment of $\mathrm{SSc}^{5}$, CCI have to be considered for first-line therapy for RP, IV prostanoids for active DU, and ET1RA (bosentan) for patients with multiple and recurring DU after failed therapy with CCI and prostanoids. Further, a published metaanalysis on healing and preventing DU revealed that PDE5 inhibitors were helpful in the healing of DU, while bosentan and iloprost may prevent new $\mathrm{DU}^{31}$ and attenuate the development of $\mathrm{PAH}^{32}$.

The lack of evidence for the benefit of pentoxifylline in patients with SSc clearly correlated with the decrease in the use of this drug between 2003 and 2013.

Table 2. Independent clinical features/risk factors associated with the use of vasoactive drugs (multivariable logistic regression). Values are OR (95\% CI).

\begin{tabular}{lcccc}
\hline Characteristics & PDE5 Inhibitors & ET1RA & ACE Inhibitors and/or AT1RA & IV Prostanoids \\
\hline Female & - & - & $0.6(0.5-0.7), \mathrm{p}<0.001$ \\
Age, yrs & - & - & $1.007(1.002-1.011), \mathrm{p}=0.006$ & - \\
RP & - & - & - & $5.1(1.2-21.4), \mathrm{p}=0.026$ \\
PAH & $7.2(4.5-11.7), \mathrm{p}<0.001$ & $6.9(4.8-9.8), \mathrm{p}<0.001$ & $1.8(1.5-2.3), \mathrm{p}<0.001$ & - \\
DU & $1.5(0.9-2.4), \mathrm{p}=0.095$ & $2.8(2.0-3.9), \mathrm{p}<0.001$ & - & $1.7(1.4-2.2), \mathrm{p}<0.001$ \\
HTN & - & - & $5.4(4.5-6.5), \mathrm{p}<0.001$ & $0.7(0.5-0.9), \mathrm{p}=0.005$ \\
\hline
\end{tabular}

PDE5: phosphodiesterase type 5; ET1RA: endothelin 1 receptor antagonists; ACE: angiotensin-converting enzyme; AT1RA: angiotensin 1 receptor antagonists; IV: intravenous; RP: Raynaud phenomenon; PAH: pulmonary arterial hypertension; DU: digital ulcers; HTN: hypertension. 
Table 3. The use of vasoactive drugs depending on different vasculopathic clinical signs and the first visit/registration of the patient (before 2005 and after 2009). There was no significant difference between the different registration periods for ACE inhibitors and PDE5 inhibitors. Values are \% unless otherwise specified.

\begin{tabular}{|c|c|c|c|}
\hline \multirow{2}{*}{ Variables } & \multicolumn{2}{|c|}{$\mathrm{RP}+/ \mathrm{DU}-/ \mathrm{PAH}-$} & \multirow[t]{2}{*}{$\mathrm{p}$} \\
\hline & $2005, \mathrm{n}=344$ & $2009, \mathrm{n}=410$ & \\
\hline All & 59.7 & 59.9 & 0.956 \\
\hline $\mathrm{CCI}$ & 61.3 & 53.2 & 0.027 \\
\hline PDE5 inhibitors & 0.6 & 1.5 & 0.302 \\
\hline ET1RA & 0.9 & 5.1 & $<0.001$ \\
\hline ACE inhibitors & 19.0 & 20.5 & 0.502 \\
\hline AT1RA & 5.5 & 13.7 & $<0.0001$ \\
\hline Pentoxifylline & 20.3 & 7.1 & $<0.0001$ \\
\hline IV prostanoids & 14.8 & 25.1 & $<0.001$ \\
\hline \multirow[t]{2}{*}{ Variables } & \multicolumn{2}{|c|}{$\mathrm{DU}+/ \mathrm{PAH}-$} & $\mathrm{p}$ \\
\hline & $2005, \mathrm{n}=141$ & $2009, \mathrm{n}=199$ & \\
\hline All & 77.5 & 75.4 & 0.661 \\
\hline $\mathrm{CCI}$ & 62.4 & 45.2 & 0.002 \\
\hline PDE5 inhibitors & 1.4 & 5.5 & 0.082 \\
\hline ET1RA & 3.5 & 19.6 & $<0.0001$ \\
\hline ACE inhibitors & 20.9 & 24.3 & 0.437 \\
\hline AT1RA & 2.8 & 7.5 & 0.092 \\
\hline Pentoxifylline & 12.8 & 11.1 & 0.733 \\
\hline IV prostanoids & 27.7 & 30.7 & 0.629 \\
\hline \multirow[t]{2}{*}{ Variables } & \multicolumn{2}{|c|}{$\mathrm{PAH}+/ \mathrm{DU}-$} & $\mathrm{p}$ \\
\hline & $2005, n=68$ & $2009, \mathrm{n}=78$ & \\
\hline All & 77.2 & 89.6 & 0.023 \\
\hline $\mathrm{CCI}$ & 45.6 & 47.4 & 0.869 \\
\hline PDE5 inhibitors & 14.7 & 16.7 & 0.822 \\
\hline ET1RA & 23.5 & 17.9 & 0.420 \\
\hline ACE inhibitors & 30.7 & 41.7 & 0.138 \\
\hline AT1RA & 10.3 & 12.8 & 0.797 \\
\hline Pentoxifylline & 26.5 & 3.8 & $<0.0001$ \\
\hline IV prostanoids & 4.4 & 23.1 & 0.002 \\
\hline
\end{tabular}

Significant data are in bold face. ACE: angiotensin-converting enzyme; PDE5: phosphodiesterase type 5; CCI: calcium channel inhibitors; ET1RA: endothelin 1 receptor antagonists; AT1RA: angiotensin 1 receptor antagonists; IV: intravenous; RP: Raynaud phenomenon; DU: digital ulcers; PAH: pulmonary arterial hypertension.

The EUSTAR has also recommended the use of bosentan or sildenafil in patients with SSc-associated PAH, and the use of ACE inhibitors in case of scleroderma renal crisis $^{5}$. Of the patients, $84.2 \%$ with PAH in our cohort were treated with vasodilating agents and of these, $24.1 \%$ were treated with ET1RA and $16.4 \%$ with PDE5 inhibitors. Interestingly, the frequency of the use of PDE5 inhibitors in patients with idiopathic PAH increased after 2006, which might be attributable to the drug approval in $2006^{33}$. Moreover, the use of ET1RA increased since 2003 after it was approved for the treatment for PAH (World Health Organization III) in 2002 and for DU in 2007. Again because of a lack of drug approval, fewer patients have been treated in Canada with ET1RA and PDE5 inhibitors ${ }^{30}$.

Our data confirm already existing treatment recommendations because (1) patients with PAH had a significantly increased chance to be treated with vasodilative drugs in general, but also to be started with ET1RA or PDE5 inhibitors, and (2) patients with DU had a 2-fold increase in the probability to be treated with vasodilative drugs, especially with ET1RA and IV prostanoids.

Unexpectedly, about one-third of patients received ACE inhibitors or AT1RA. This reflects the use of this drug class as first-line therapy in patients with HTN and/or proteinuria ${ }^{5}$. Further, although overall frequency of vasoactive therapy did not differ between rheumatologists and dermatologists, significant differences were found for the drugs used. Of these, significantly more patients reviewed by rheumatologists received ET1RA and PDE5 inhibitors compared with patients seen by dermatologists who were treated significantly more often with IV prostanoids.

To evaluate whether treatment recommendations change 


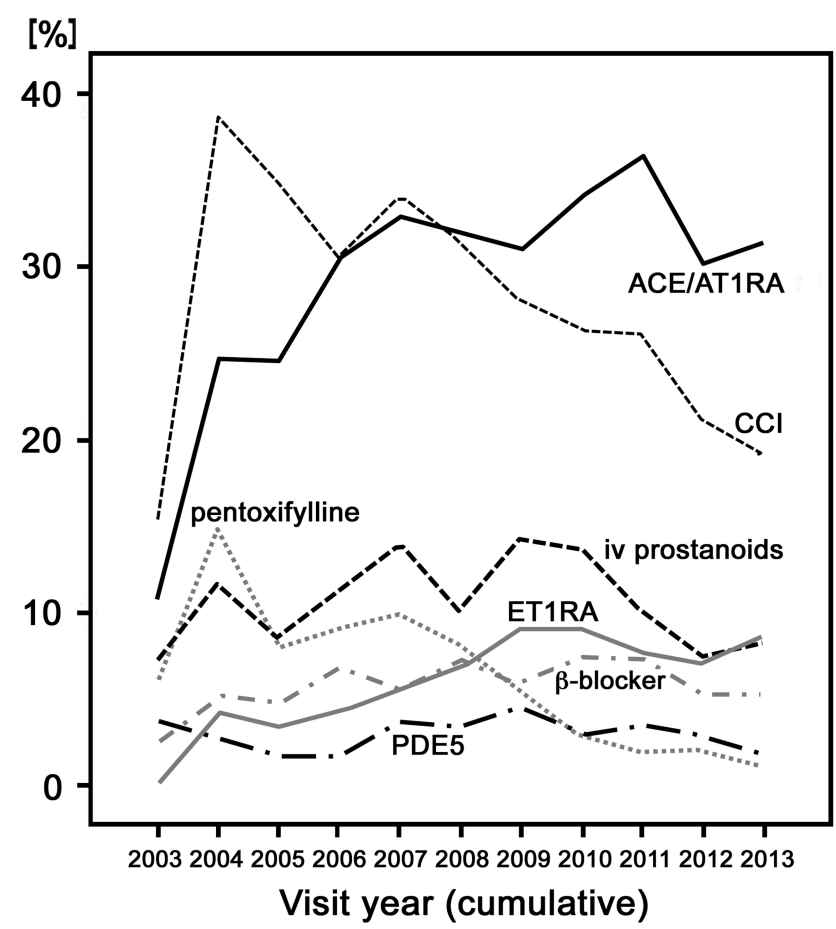

Figure 2. The frequency of the prescription of different vasodilative agents depending on the year of the visit. ACE: angiotensin-converting enzyme; AT1RA: angiotensin 1 receptor antagonists; CCI: calcium channel inhibitors; IV: intravenous; ET1RA: endothelin 1 receptor antagonists; PDE5: phosphodiesterase type 5 .

the way and frequency of treatment strategies, we compared patients who were registered prior to 2005 and those registered after 2009. The comparison revealed a significant increase of patients who were treated with vasodilative drugs, especially for patients with RP. The frequency of patients treated with ET1RA, AT1RA, and IV prostanoids has increased significantly, while the use of pentoxifylline has decreased significantly (Table 2). This is also true for the use of ET1RA in patients with DU ( $<<0.001)$, which is certainly associated with the first reports about the preventive effect of bosentan in patients with DU around $2004^{10,34}$. IV prostanoids were clearly more often used after 2009 ( $\mathrm{p}<$ $0.001)$ and especially in those patients presenting with RP $(\mathrm{p}=0.001)$ and PAH $(\mathrm{p}=0.002)$, but interestingly no significant raise was detectable in patients with DU (intermittent use). Altogether, management of patients with vasculopathies has considerably improved compared with the status when patients were registered in the first years of DNSS. At the time, CCI were the first-line therapy in most centers, but often applied in insufficient dosages, and only $21.2 \%$ of patients with DU had received prostacyclins ${ }^{35}$. These data show that the standardized management and treatment recommendations have reached clinical practice and improved management of patients with SSc and vasculopathy.

A number of limitations have to be considered for this study. SSc is a multisystem disease and different organ involvements are induced by vascular involvement. Therefore, a patient with RP and DU may receive 2 vasoactive agents (i.e., CCI and ET1RA, etc.). The questionnaire, for the sake of feasibility, however, is not able to identify which drug has been used for which symptom. This also holds true for the data identification of comorbid disorders, side effects, and average dosage and duration of the treatment used, as well as information on improvement or aggravation of symptoms after initiation of treatment.

Our study shows that many patients with SSc with signs of vasculopathy do not yet receive sufficient vasoactive treatment. Nevertheless, the introduction of new classes of vasoactive drugs in recent years has significantly improved both treatment recommendations and options in clinical practice.

\section{APPENDIX 1.}

List of study collaborators. German Network for Systemic Scleroderma (DNSS): Nil Mona Ahrazoglu, Doreen Belz, Department of Dermatology, Cologne University Hospital; Mike Oliver Becker, Department of Rheumatology and Clinical Immunology, University of Berlin, Charité; Ingo Tarner, Department of Rheumatology and Clinical Immunology, Kerckhoff Clinic, Bad Nauheim; Rudolf Mierau, Department of Rheumatology, Clinic of Rheumatology Aachen; Andrea Rubbert, Department of Rheumatology, University of Cologne; Rebecca Fischer-Betz, Department of Nephrology and Rheumatology, University of Duesseldorf; Ivan Foeldvári, Department of Rheumatology, Schoen Clinic, Hamburg-Eilbek; Johannes Norgauer, Department of Dermatology and Venereology, University of Jena; Dieter Schoeffel, Clinic for Rheumatology, Mannheim; Regina Renner, Mirjana Ziemer, Department of Dermatology and Venereology, University of Leipzig; Rudolf Stadler, Verena Müller, Department of Dermatology and Venereology, Johannes Wesling Hospital, Minden; Mahzad Ziai, Department of Dermatology, Munich University of Technology; Michael Landthaler, Department of Dermatology and Venereology, University of Regensburg; Rotraud Meyringer, Department of Internal Medicine, University of Regensburg; Dirk Tomsitz, Hae-Hyuk Lee, Department of Dermatology and Venereology, University of Berlin, Charité; Theo Xenitidis, Department of Rheumatology, University of Tuebingen; Joachim Ebel, Department of Rheumatology, Asklepios Clinic Altona; Christian Beyer, Department of Rheumatology, University of Erlangen; Tina van Eijk, Georg Mitrakos, Department of Dermatology, HELIOS St. Elisabeth Clinic Oberhausen; Nora Wutte, Department of Dermatology, Medical University of Graz; Frank Reichenberger, Department of Pneumology, Asklepios Clinic Gauting, Muenchen-Gauting, Lena Herich, Institute of Medical Statistics, Informatics and Epidemiology, University of Cologne, Germany; Michael P. Schoen, Department of Dermatology and Venerology, University of Goettingen, Germany.

\section{ACKNOWLEDGMENT}

The work of Ebru Keser, Patricia Scholz, and Gabriele Browne in keeping the Network going at the central office is gratefully acknowledged.

\section{REFERENCES}

1. Guiducci S, Giacomelli R, Cerinic MM. Vascular complications of scleroderma. Autoimmun Rev 2007;6:520-3.

2. Galluccio F, Matucci-Cerinic M. Two faces of the same coin: Raynaud phenomenon and digital ulcers in systemic sclerosis. Autoimmun Rev 2011;10:241-3.

3. Gabrielli A, Avvedimento EV, Krieg T. Scleroderma. N Engl J Med 2009;360:1989-2003.

4. Walker KM, Pope J; participating members of the Scleroderma Personal non-commercial use only. The Journal of Rheumatology Copyright @ 2017 . All rights reserved. 
Clinical Trials Consortium (SCTC); Canadian Scleroderma Research Group (CSRG). Treatment of systemic sclerosis complications: what to use when first-line treatment fails - a consensus of systemic sclerosis experts. Semin Arthritis Rheum 2012;42:42-55.

5. Kowal-Bielecka O, Landewé R, Avouac J, Chwiesko S, Miniati I, Czirjak L, et al; EUSTAR Co-Authors. EULAR recommendations for the treatment of systemic sclerosis: a report from the EULAR Scleroderma Trials and Research group (EUSTAR). Ann Rheum Dis 2009;68:620-8

6. Walker KM, Pope J; Scleroderma Clinical Trials Consortium; Canadian Scleroderma Research Group. Expert agreement on EULAR/EUSTAR recommendations for the management of systemic sclerosis. J Rheumatol 2011;38:1326-8.

7. Riemekasten G, Sunderkötter C. Vasoactive therapies in systemic sclerosis. Rheumatology 2006;45 Suppl 3:iii49-51.

8. Riemekasten G, Hoffmann U, Sunderkotter C, Weiss N, Kuhn A; angiologisch-dermatologisch-rheumatologische DU-Expertenboard. [Management of digital ulcers in patients with systemic sclerosis]. [Article in German] Dtsch Med Wochenschr 2012;137:34-40.

9. Thompson AE, Shea B, Welch V, Fenlon D, Pope JE. Calcium-channel blockers for Raynaud's phenomenon in systemic sclerosis. Arthritis Rheum 2001;44:1841-7.

10. Korn JH, Mayes M, Matucci Cerinic M, Rainisio M, Pope J, Hachulla E, et al. Digital ulcers in systemic sclerosis: prevention by treatment with bosentan, an oral endothelin receptor antagonist. Arthritis Rheum 2004;50:3985-93.

11. Sitbon O, Badesch DB, Channick RN, Frost A, Robbins IM, Simonneau G, et al. Effects of the dual endothelin receptor antagonist bosentan in patients with pulmonary arterial hypertension: a 1-year follow-up study. Chest 2003;124:247-54 .

12. Matucci-Cerinic M, Seibold JR. Digital ulcers and outcomes assessment in scleroderma. Rheumatology 2008;47 Suppl 5:v46-7.

13. Hachulla E, Denton CP. Early intervention in pulmonary arterial hypertension associated with systemic sclerosis: an essential component of disease management. Eur Respir Rev 2010; 19:314-20.

14. McLaughlin V, Humbert M, Coghlan G, Nash P, Steen V. Pulmonary arterial hypertension: the most devastating vascular complication of systemic sclerosis. Rheumatology 2009;48 Suppl 3:iii25-31.

15. Dhillon S. Macitentan: a review of its use in patients with pulmonary arterial hypertension. Drugs 2014;74:1495-507.

16. Steen V, Denton CP, Pope JE, Matucci-Cerinic M. Digital ulcers: overt vascular disease in systemic sclerosis. Rheumatology 2009;48 Suppl 3:iii19-24.

17. Wigley FM, Seibold JR, Wise RA, McCloskey DA, Dole WP. Intravenous iloprost treatment of Raynaud's phenomenon and ischemic ulcers secondary to systemic sclerosis. J Rheumatol 1992;19:1407-14.

18. Wigley FM, Wise RA, Seibold JR, McCloskey DA, Kujala G, Medsger TA Jr, et al. Intravenous iloprost infusion in patients with Raynaud phenomenon secondary to systemic sclerosis. A multicenter, placebo-controlled, double-blind study. Ann Intern Med 1994;120:199-206.

19. Chrysant SG. Vascular remodeling: the role of angiotensin-converting enzyme inhibitors. Am Heart J 1998;135:S21-30.

20. Dziadzio M, Denton CP, Smith R, Howell K, Blann A, Bowers E, et al. Losartan therapy for Raynaud's phenomenon and scleroderma: clinical and biochemical findings in a fifteen-week, randomized, parallel-group, controlled trial. Arthritis Rheum 1999;42:2646-55.

21. Whitman HH 3rd, Case DB, Laragh JH, Christian CL,
Botstein G, Maricq H, et al. Variable response to oral angiotensin-converting-enzyme blockade in hypertensive scleroderma patients. Arthritis Rheum 1982;25:241-8.

22. Brueckner CS, Becker MO, Kroencke T, Huscher D, Scherer HU, Worm M, et al. Effect of sildenafil on digital ulcers in systemic sclerosis: analysis from a single centre pilot study. Ann Rheum Dis 2010;69:1475-8.

23. Kumar U, Sankalp G, Sreenivas V, Kaur S, Misra D. Prospective, open-label, uncontrolled pilot study to study safety and efficacy of sildenafil in systemic sclerosis-related pulmonary artery hypertension and cutaneous vascular complications. Rheumatol Int 2013;33:1047-52.

24. Fries R, Shariat K, von Wilmowsky H, Böhm M. Sildenafil in the treatment of Raynaud's phenomenon resistant to vasodilatory therapy. Circulation 2005;112:2980-5.

25. Roustit M, Blaise S, Allanore Y, Carpentier PH, Caglayan E, Cracowski JL. Phosphodiesterase-5 inhibitors for the treatment of secondary Raynaud's phenomenon: systematic review and meta-analysis of randomised trials. Ann Rheum Dis 2013; 72:1696-9.

26. Hunzelmann N, Genth E, Krieg T, Lehmacher W, Melchers I, Meurer M, et al; Registry of the German Network for Systemic Scleroderma. The registry of the German Network for Systemic Scleroderma: frequency of disease subsets and patterns of organ involvement. Rheumatology 2008;47:1185-92.

27. Hunzelmann N, Moinzadeh P, Genth E, Krieg T, Lehmacher W, Melchers I, et al; German Network for Systemic Scleroderma Centers. High frequency of corticosteroid and immunosuppressive therapy in patients with systemic sclerosis despite limited evidence for efficacy. Arthritis Res Ther 2009;11:R30.

28. Moinzadeh P, Aberer E, Ahmadi-Simab K, Blank N, Distler JH, Fierlbeck G, et al; all participating DNSS centers. Disease progression in systemic sclerosis-overlap syndrome is significantly different from limited and diffuse cutaneous systemic sclerosis. Ann Rheum Dis 2015;74:730-7.

29. Opitz C, Klein-Weigel PF, Riemekasten G. Systemic sclerosis - a systematic overview: part 2 - immunosuppression, treatment of SSc-associated vasculopathy, and treatment of pulmonary arterial hypertension. Vasa 2011;40:20-30.

30. Pope J, Harding S, Khimdas S, Bonner A, Baron M; Canadian Scleroderma Research Group. Agreement with guidelines from a large database for management of systemic sclerosis: results from the Canadian Scleroderma Research Group. J Rheumatol 2012;39:524-31.

31. Tingey T, Shu J, Smuczek J, Pope J. Meta-analysis of healing and prevention of digital ulcers in systemic sclerosis. Arthritis Care Res 2013;65:1460-71.

32. Romaniello A, Viola G, Salsano F, Rosato E. In systemic sclerosis patients, bosentan is safe and effective for digital ulcer prevention and it seems to attenuate the development of pulmonary arterial hypertension. Rheumatology 2014;53:570-1.

33. Kane LB, Klings ES. Present and future treatment strategies for pulmonary arterial hypertension: focus on phosphodiesterase-5 inhibitors. Treat Respir Med 2006;5:271-82.

34. Matucci-Cerinic M, Denton CP, Furst DE, Mayes MD, Hsu VM, Carpentier P, et al. Bosentan treatment of digital ulcers related to systemic sclerosis: results from the RAPIDS-2 randomised, double-blind, placebo-controlled trial. Ann Rheum Dis 2011; 70:32-8.

35. Herrgott I, Riemekasten G, Hunzelmann N, Sunderkötter C. Management of cutaneous vascular complications in systemic scleroderma: experience from the German network. Rheumatol Int 2008;28:1023-9. 